\title{
Arginine Feeding Modifies Cyclosporine Nephrotoxicity in Rats
}

\author{
Luca De Nicola, Scott C. Thomson, Lucinda M. Wead, Marvin R. Brown, and Francis B. Gabbai \\ Division of Nephrology-Hypertension, Department of Medicine, Veterans Affairs Medical Center and University of California, \\ San Diego School of Medicine, La Jolla, California 92161
}

\begin{abstract}
Glycine ( $G$ ) infusion causes renal vasodilation mediated by nitric oxide (NO). Cyclosporine A (CsA) nephrotoxicity is characterized by preglomerular vasoconstriction and decreased efferent arteriolar tone probably related to reduced NO and angiotensin II, respectively. L-Arginine (ARG) is a precursor to NO. To test the hypothesis that chronic CsA decreases renal NO activity, we compared the glomerular hemodynamic response to glycine infusion in rats after $8 \mathrm{~d}$ of $\mathrm{CsA}(30 \mathrm{mg} / \mathrm{kg}$ per $\mathrm{d}$ s.c.), CsA and ARG (1.6 g/kg per d p.o.) (A/CsA), and in two groups of pair-fed controls (CON, A/CON). Single nephron GFR (SNGFR), single nephron plasma flow (SNPF), glomerular capillary hydrostatic pressure gradient $(\Delta P)$, proximal tubular reabsorption (APR), and kidney tissue angiotensin II (AIIk) were measured before and during G. CsA was associated with baseline decrements in SNGFR, SNPF, $\triangle P$, and AIIk, and with a blunted hemodynamic response to $G$. In $\mathrm{CON}$, ARG did not affect baseline hemodynamics or modify the response to G. In CsA, ARG decreased baseline preglomerular resistance and restored the glomerular hemodynamic response to $G$. $G$ was associated with a significant increase in AIIk in both CON and CsA. These findings suggest that (a) CsA is associated with decreased AIIk, and (b) CsA may diminish NO activity within the kidney, and that this capacity may be partially restored by arginine feeding. (J. Clin. Invest. 1993. 92:1859-1865.) Key words: angiotensin II $\bullet$ L-arginine $\bullet$ cyclosporine $\bullet$ glycine $\bullet$ nitric oxide
\end{abstract}

\section{Introduction}

While of undeniable usefulness in clinical medicine, the administration of cyclosporine $\mathrm{A}$ ( CsA $)^{1}$ is encumbered by deleterious effects on renal function (1). Much research has been undertaken in this area (2-7), but the pathophysiologic mechanisms underlying CsA nephrotoxicity remain to be fully

Portions of this work were presented at the 24th Meeting of the American Society of Nephrology, Baltimore, 1991 and have been published in abstract form (1991. J. Am. Soc. Nephrol. 2:781).

Address reprint requests to Dr. Francis Gabbai, Division of Nephrology-Hypertension 9111-H, Veterans Affairs Medical Center, 3350 La Jolla Village Drive, San Diego, CA 92161.

Received for publication 26 January 1993 and in revised form 6 May 1993.

1. Abbreviations used in this paper: AII, angiotensin II; APR, absolute proximal reabsorption; CsA, cyclosporine A; EFP, effective filtration pressure; FR, fractional reabsorption; L-NMMA, ${ }^{G}$-monomethyl-Larginine; LpA, glomerular ultrafiltration coefficient; MAP, mean arterial pressure; NO, nitric oxide; TGF, tubuloglomerular feedback.

The Journal of Clinical Investigation, Inc.

Volume 92, October 1993, 1859-1865 elucidated. One contemporary hypothesis invokes CsA-mediated endothelial damage as a direct antecedent to glomerular hypoperfusion. Indeed, CsA is toxic to endothelial cells in vitro $(8)$, and preliminary reports suggest impaired endotheliumdependent relaxation in renal and systemic vessels after prolonged CsA exposure (9-11). It is plausible, therefore, that endothelial dysfunction with reduced activity of the endothelial-derived relaxing factor, nitric oxide (NO), might underlie the functional effect of CsA on glomerular filtration.

Other recent evidence indicates that NO interacts with angiotensin II (AII) to govern glomerular hemodynamics and tubular function in normal rats (12). Endogenous NO attenuates the glomerular effects of AII and is required to permit normal proximal tubular reabsorption. Furthermore, NO mediates an increase in GFR during amino acid infusion, with glomerular and tubular responses to amino acid loading dependent on the balance between NO and AII (12). Therefore, it is reasonable to employ the renal response to infusion of the amino acid glycine to test the capacity of the kidney to generate $\mathrm{NO}$ and to assess the state of NO/AII balance in vivo.

The role of the renin-angiotensin system in CsA nephrotoxicity remains controversial and probably varies with the duration of CsA administration $(6,13-15)$. Data from this laboratory have demonstrated that short-term chronic CsA administration leads to an imbalance between pre- and postglomerular resistances and a decrease in effective filtration pressure, a scenario compatible with a diminished AII response (6). Because afferent arteriolar resistance is influenced by several mediators, while efferent arteriolar resistance is governed primarily by AII, the functional effects of chronically administered CsA on the kidney may involve an inadequate efferent arteriolar AII response to preglomerular vasoconstriction. This hypothesis has not been tested by direct measurement of intrarenal AII concentrations.

Chronic ingestion of $\mathrm{L}$-arginine reduces blood pressure in hypertensive rats (16-18) and delays the progression of renal disease in rats with remnant kidneys (19) by mechanisms which remain undefined. In that $\mathrm{L}$-arginine is a substrate for the synthesis of NO (20), the salutary influence of L-arginine on blood pressure and renal function may be mediated by NO.

We therefore assessed the response to glycine infusion in the CsA nephrotoxic model in the rat and the capacity of arginine to potentially normalize the response to glycine in order to (a) evaluate the glomerular and tubular responses to glycine infusion in CsA treated rats, $(b)$ determine the potential for L-arginine feeding to modify the response to glycine, and $(c)$ estimate the impact of CsA and L-arginine on intrarenal AII by direct measurement.

\section{Methods}

Experiments were conducted in 53 male Munich-Wistar rats (180-220 g body wt) obtained from Simonsen Laboratories (Gilroy, CA). Animals were allowed free access to tap water. During the 8-d protocol 
period, rats treated with CsA and their pair-fed counterparts ate 16 $\mathrm{g} / 24 \mathrm{~h}$ of standard rat laboratory diet. This dietary protocol was chosen on the basis of our previous report (6) and preliminary studies showing rats undergoing CsA treatment eat an average of $16 \mathrm{~g} / \mathrm{d}$. Four groups of rats were studied. Group CsA, $(n=13)$ received CsA (Sandoz Pharmaucetical Morris Plains, $\mathrm{NJ}$ ) at the dose of $30 \mathrm{mg} / \mathrm{kg}$ body wt administered in olive oil by daily subcutaneous injection for eight consecutive days before micropuncture. Group A/CsA $(n=17)$, received parenteral CsA and oral L-arginine $(1.6 \mathrm{~g} / \mathrm{kg}$ per d $)$ mixed with their normal diet. Similar doses of $\mathrm{L}$-arginine in the drinking water reduce blood pressure in hypertensive rats (16). Pair-fed control groups, CON ( $n$ $=12)$ and $\mathrm{A} / \mathrm{CON}(n=12)$, were injected with olive oil, $\mathrm{A} / \mathrm{CON}$ also receiving $\mathrm{L}$-arginine in a dose similar to $\mathrm{A} / \mathrm{Cs} \mathrm{A}$ group.

\section{Micropuncture studies}

Seven rats in group CsA, seven rats in group A/CsA, six rats in group $\mathrm{CON}$, and six rats in group $\mathrm{A} / \mathrm{CON}$ underwent micropuncture. The animals were allowed free access to food and tap water until the morning of the experiment.

Micropuncture was performed according to protocols previously described in publications from this laboratory $(21,22)$. After Inactin (BYK, Constance, FRG) anesthesia (100 mg/kg i.p.) and tracheostomy (PE 240), catheters (PE 50) were placed in the right jugular vein, left femoral artery, urinary bladder, and left ureter. Body temperature was regulated by a servocontrolled heating table. Euvolemic conditions were reconstituted with donor rat plasma as surgical replacement ( 1 $\mathrm{ml} / 100 \mathrm{~g}$ body wt over $60 \mathrm{~min}$, followed by continuous infusion, 0.15 $\mathrm{ml} / 100 \mathrm{~g}$ body $\mathrm{wt} / \mathrm{h}$ ). All animals also received Ringers' saline containing $140 \mu \mathrm{Ci} / \mathrm{ml}\left[{ }^{3} \mathrm{H}\right]$ inulin $(0.8 \mathrm{ml} / \mathrm{h})$ as a marker of glomerular filtration.

Arterial blood pressure was monitored from the femoral artery catheter via a P23dB Statham pressure transducer and Statham chart recorder (Gould Inc., Glen Burnie, MD). Hydrostatic pressures were measured directly in glomerular capillaries $\left(P_{\mathrm{GC}}\right)$, urinary space $\left(P_{\mathrm{US}}\right)$, and efferent arterioles $\left(\mathrm{HP}_{\mathrm{E}}\right)$ with a servonulling pressure device employing micropipettes filled with hypertonic saline. Systemic blood was sampled from the femoral artery. Efferent arteriolar blood was obtained by direct micropuncture. A microadaptation of the Lowry technique (23) was employed to determine the protein concentrations of systemic $\left(C_{\mathrm{A}}\right)$ and efferent $\operatorname{arteriolar}\left(C_{\mathrm{E}}\right)$ plasma. Plasma oncotic pressure $(\pi)$ was calculated from protein concentration by the Landis-Pappenheimer equation (24). Nephron filtration fraction was computed from $C_{\mathrm{A}}$ and $C_{\mathrm{E}}$. Plasma, tubular fluid, and urine were assayed for $\left[{ }^{3} \mathrm{H}\right]$ inulin concentration with a liquid scintillation counter (model B4530 Tri/Carb, Packard Instruments, Inc., Downers Grove, IL). Inulin clearance and volumetric measurement of fluid collected from late proximal tubules were employed to calculate single nephron (SN) GFR and late proximal flow rate $\left(V_{L P}\right)$. Absolute $(A P R)$ and fractional (FR) rates of proximal reabsorption were calculated from SNGFR and $\mathrm{V}_{\mathrm{LP}}$. GFR was calculated as urinary inulin clearance.

Two-period studies were employed to test the response to glycine in each animal. During the first, or baseline (B) period, each animal received an additional infusion of Ringers' saline $(1.4 \mathrm{ml} / \mathrm{h})$. During a 30-min reequilibration period and throughout the second experimental period each animal received glycine $(G)$ ( $2.66 \mathrm{M}$ in Ringers' saline at $1.4 \mathrm{ml} / \mathrm{h}$ ). Data collected during each experimental period involved pressure measurements from three glomeruli and three efferent arterioles, two systemic and three efferent arteriolar plasma samples, and five tubular fluid collections. Measurements and samplings were performed within a span of $60 \mathrm{~min}$.

To investigate if arginine feeding modified the glomerular and tubular response to glycine through a NO-dependent mechanism, the effect of the NO synthase inhibitor $\mathrm{N}^{\mathrm{G}}$ monomethyl L-arginine ( $\mathrm{L}$ NMMA) was evaluated in four additional A/CsA rats. SNGFR and APR were measured during baseline period and during the simultaneous infusion of glycine $(2.66 \mathrm{M}$ in Ringer's at $1.4 \mathrm{ml} / \mathrm{h})$ plus $\mathrm{L}$ NMMA $(0.5 \mathrm{mg} / \mathrm{kg}$ per $\mathrm{min})$.

\section{Determination of plasma and renal angiotensin II levels}

Plasma and renal tissue angiotensin II concentrations were measured in samples obtained after micropuncture and from additional Inactinanesthetized rats pretreated with $\mathrm{CsA}$ and arginine, but not subjected to micropuncture.

Plasma processing. $1.0 \mathrm{ml}$ of blood was collected in a syringe containing $20 \mu \mathrm{l}$ of EDTA $(0.16 \mathrm{M})$ and $10 \mu$ l of converting enzyme inhibitor $(0.1 \mathrm{mM})$. Samples were spun at $4^{\circ} \mathrm{C}$ and plasma stored at $-70^{\circ} \mathrm{C}$ until processed. Plasma was extracted using a Bondelut $\mathrm{C}_{18}$ column previously washed with methanol and triethylamine-formic acid. The column was rinsed with triethylamine formic acid buffer and AII was eluted off with acetonitrile triethylamine formic acid 70:30, lyophilized on a Speed-Vac overnight, and kept at $-20^{\circ} \mathrm{C}$ until assayed. This extraction procedure yielded $92 \%$ recovery of AII.

Renal tissue processing. Kidneys were perfused free of blood with $50 \mathrm{ml}$ of a solution containing $4.9 \mathrm{mM}$ 8-hydroxyquinoline hemisulfate, $2.6 \mathrm{mM}$ EDTA and 3\% BSA administered through an aortic catheter. Bloodless kidneys were excised, placed in a plastic container, flash-frozen in liquid nitrogen, and stored at $-70^{\circ} \mathrm{C}$ until further processing. Individual whole kidneys were homogenized (Polytron, Brinkmann Instruments Inc., Westbury, NY; 10 at setting 6) in $2 \mathrm{ml}$ of RIA-BSA $0.25 \%$ buffer, added to $9 \mathrm{ml}$ of homogenizing medium ( $1 \mathrm{~N}$ glacial acetic acid, $0.02 \mathrm{~N}$ hydrochloric acid, and $0.1 \% 2$ mercaptoethanol), and heated to $90^{\circ} \mathrm{C}$ for $10 \mathrm{~min}$, then centrifuged at $30,000 \mathrm{~g}$ for $20 \mathrm{~min}$. The supernatant, $\mathrm{s}_{1}$, was removed and the pellet resuspended in $4.5 \mathrm{ml}$ of homogenizing medium and centrifuged at $30,000 \mathrm{~g}$ for 20 min. The supernatant was combined with $s_{1}$, and lyophilized overnight using a Speed Vac centrifuge (model RH200-12, Savant Instruments Inc., Farmingdale, NY). The resulting lyophylisate was resuspended in $2 \mathrm{ml}$ of RIA-BSA $0.25 \%$ buffer and $500 \mu$ l of this extracted using a Bondelut $\mathrm{C}_{18}$ column as described above for plasma. This combined procedure yielded $56 \%$ recovery of AII.

$R I A$. Lyophylisate was resuspended in $500 \mu$ l of RIA buffer and 200 $\mu l$ of this was added to $100 \mu$ l of specific rabbit All-antibody (Ab 127, provided by Dr. Wylie Vale, Salk Institute, La Jolla, CA) diluted $1 / 62,500$. After incubating for $2 \mathrm{~d}$ at $4^{\circ} \mathrm{C}, 100 \mu \mathrm{l}$ of ${ }^{125} \mathrm{I}$-AII $(6,000$ cpm) (New England Nuclear, Boston, MA), were added to each tube and incubated again at $4^{\circ} \mathrm{C}$ overnight. AII bound to antibody was separated from unbound using normal rabbit serum diluted 1/200 (100 $\mu \mathrm{l})$, goat anti-rabbit IgG diluted $1 / 40(100 \mu \mathrm{l})$ and $500 \mu \mathrm{l}$ of $10 \%$ polyethylene glycol in RIA buffer added to each tube. Tubes were incubated at $4^{\circ} \mathrm{C}$ for $2 \mathrm{~h}$ and then spun for $30 \mathrm{~min}$, the supernatant was decanted, and the pellet was counted in a gamma counter (Searle model 1185, T. M. Analytic, Elk Grove Village, IL, $80 \%$ efficiency). AII concentrations were calculated using a computer aided logit/log transformation of the standard curve. Cross-reactivity of this AII antibody with angiotensin I is $0.33 \%$ and with angiotensin III is $68 \%$. Plasma and kidney samples were equally divided in four different assays. Interassay error was $15 \%$.

HPLC. A Beckman (altex) 450 Data/System controller with a 210 variable $\lambda$ detector and two $114 \mathrm{M}$ pumps were utilized. The altex $210 \mathrm{~A}$ injector valve was fitted with a 500- $\mu$ l sample loop. Solution A was $0.1 \%$ trifluoracetic acid in HPLC grade water. Solution B was $80 \%$ acetonitrile, $0.1 \%$ trifluoroacetic acid. For HPLC, lyophilized tissue extracts estimated to contain $250 \mathrm{pg}$ of AII, $250 \mathrm{pg}$ of synthetic AII (Sigma Laboratories, St Louis, MO), or tissue extracts with added synthetic AII (total $500 \mathrm{pg}$ ) was reconstituted in $500 \mu \mathrm{l} 20 \%$ solution B. A $500-\mu$ l quantity of each was injected into a Vydac $C_{18} 5-\mu \mathrm{m}$ reversephase column equilibrated to $20 \%$ solution B. A 20 -min gradient was run to $40 \%$ solution $B$ followed by isocratic run for $7 \mathrm{~min}$. Each run was preceded and followed by a blank gradient to elute any material bound to the column from the previous run. The flow rate was $1 \mathrm{ml} / \mathrm{min}$ and 1 -min fractions were collected in polypropylene tubes containing $0.1 \%$ BSA. The tubes were lyophilized and maintained at $-20^{\circ} \mathrm{C}$ until RIA. All samples (synthetic AII, kidney tissue and kidney tissue + AII) eluted with a similar pattern with a peak maximum activity at $15 \mathrm{~min}$. Recovery was $45 \%$ for synthetic AII, $52 \%$ for CON, $59 \%$ for CSA, $43 \%$ in CON + synthetic AII, and $41 \%$ for CSA + synthetic AII. 


\section{Calculations}

The determinants of SNGFR are as follows $(21,25):(a)$ single nephron plasma flow, $\mathrm{SNPF} \equiv\left(\mathrm{SNGFR}{ }^{*} C_{\mathrm{E}} /\left(C_{\mathrm{E}}-C_{\mathrm{A}}\right)\right] ;(b)$ afferent effective filtration pressure, $\mathrm{EFP}_{\mathrm{A}} \equiv \Delta P-\pi_{\mathrm{A}} ;$ and $(c)$ glomerular ultrafiltration coefficient, LpA, such that;

$\mathrm{LpA}=\frac{\text { SNGFR }}{\int_{0}^{1} \operatorname{EFP}(x) \cdot \mathrm{d} x}$

where $\mathbf{x}$ is the axial position along a nondimensionalized glomerular capillary,

$$
\begin{aligned}
& \operatorname{EFP}(x)=\Delta P(x)-\pi(x) \\
& \pi(x)=1.73 \cdot C(x)+0.28 \cdot c^{2}(x)
\end{aligned}
$$

and the plasma protein concentration, $C(x)$, is calculated according to standard formulas with the boundary conditions $C(0)=C_{\mathrm{A}}$ and $C(1)$ $=C_{\mathrm{E}}$. Additionally, afferent $\left(A_{\mathrm{R}}\right)$ and efferent $\left(E_{\mathrm{R}}\right)$ arteriolar resistances were calculated from MAP, $P_{\mathrm{G}}, \mathrm{HP}_{\mathrm{E}}, \mathrm{Hct}, \mathrm{CA}, \mathrm{CE}$, and SNGFR according to Ohm's law.

\section{Statistical analysis}

For parameters measured once during an experimental period, the effects of treatments for all two-period studies were analyzed by ANOVA with design for repeated measures (26). For parameters measured more than once during an experimental period, (SNGFR, APR, FR, $P_{\mathrm{G}}, P_{\mathrm{US}}, \mathrm{HP}_{\mathrm{E}}$, and $\Delta P$ ) effects were analyzed by standard three-way ANOVA using individual measurements. In the subsequent text, mention of the "main effect" of a treatment refers to the main effect, by ANOVA, on groups receiving that treatment. Student's $t$ test was also applied to certain intergroup comparisons as appropriate. All results are expressed as mean \pm SEM and statistical significance was defined as $P<0.05$.

\section{Results}

Body weight at the start of pair-feeding did not differ between groups $(230 \pm 5,244 \pm 8,219 \pm 13$, and $240 \pm 6 \mathrm{~g}$ for CsA, A/CsA, $\mathrm{CON}$, and $\mathrm{A} / \mathrm{CON}$, respectively). Body weight did not change significantly during the $8 \mathrm{~d}$ of treatment in any group (weight at micropuncture $224 \pm 4,235 \pm 5,222 \pm 6$, and $232 \pm 3 \mathrm{~g}$ for CsA, $\mathrm{A} / \mathrm{CsA}, \mathrm{CON}$, and $\mathrm{A} / \mathrm{CON}$, respectively).

Effects of CsA, L-arginine, and glycine infusion on glomerular hemodynamics. Micropuncture results are reported in Table I. CsA exerted several significant main effects, provoking decrements in blood pressure, GFR, SNGFR, SNPF, PG, $\Delta P$, and increments in pre- and postglomerular vascular resistances. The effect of CsA on SNGFR was mediated by a major reduction in effective filtration pressure and a minor reduction in SNPF, both of which appeared to result from a failure of afferent arteriolar autoregulatory vasodilation. The primary effects of CsA on AR, and subsequently, $\Delta P$ and EFP, were attenuated by treatment with $\mathrm{L}$-arginine. Administration of L-arginine reduced afferent arteriolar resistance in CsA $(P<0.05)$, but not CON animals, an effect which could not be linked to autoregulation. The coexistence of lower afferent resistance, unmodified blood pressure, and decreased $P_{\text {Us }}$ in A/CsA vs. CsA rats resulted in a near-doubling of EFP. Although neither CsA nor ARG exerted significant main effects on the ultrafiltration coefficient, there was a tendency for ARG to reduce LpA among the subset of animals receiving CsA. As a result, ARG did not condition the effect of CsA on SNGFR. The administration of ARG to control rats had no discernible effect on any of the measured parameters.

Significant main effects of glycine infusion (G) included increments in MAP, GFR, SNGFR, SNPF, and EFP, and decreases in Hct, $\pi_{A}$, and ER. Pretreatment with CsA significantly enhanced the effect of glycine on MAP but attenuated the effect of glycine on GFR and obliterated the effect of glycine on SNGFR. The mitigating influence of CsA on the response of glomerular filtration to glycine resulted from the sum of interactions between CsA and glycine in determining SNPF and $\mathrm{LpA}$, although large variance in SNPF among CsA-G rats and in $\mathrm{LpA}$ among CsA-B rats prevented these interactions from achieving statistical significance by ANOVA.

Co-treatment with ARG in rats receiving CsA restored the responsiveness of GFR and SNGFR to glycine, with the filtration response to $G$ among $A / C s A$ rats similar to that of $C O N$ animals. The mechanisms by which arginine restored the response of SNGFR to glycine among animals receiving CsA involved a sum of effects on the reactions of SNPF and LpA to glycine, although again, the individual interactions were not statistically significant. The vasodilatory response to glycine observed in control animals as manifested by a decrease in AR may have been masked by the autoregulatory response to the increase in MAP associated with glycine in rats treated with CsA and ARG.

Proximal tubular reabsorption before and during glycine infusion. Among the main effects of CsA were a significant increase in fractional reabsorption (FR) within the proximal tubule and an insignificant decrease in absolute reabsorption (APR) from the same segment. Neither of these effects were conditioned by L-arginine, which did not singularly influence either index of proximal reabsorption. Glycine significantly depressed FR while tending to increase APR overall. Pretreatment with CsA, however, significantly altered the proximal tubular response to glycine infusion, promoting a decrement in FR and causing an actual decline in APR. This effect of CsA on the tubular response to glycine was attenuated by co-administration of $\mathrm{L}$-arginine, which, as mentioned above, had no direct effect on proximal reabsorption, and did not interact directly with $\mathrm{G}$.

Effect of L-NMMA administration. The effect of $\mathrm{G}$ on SNGFR among A/CsA rats was blocked by prior administration of L-NMMA ( $31 \pm 3$ vs. $30 \pm 3 \mathrm{nl} / \mathrm{min})$. In this group, $G$ caused APR to decrease $(14 \pm 1$ to $10 \pm 1 \mathrm{nl} / \mathrm{min}, P<0.05)$. These results suggest that the effects of arginine feeding in CsA rats are mediated by increases in NO.

Plasma and intrarenal angiotensin II levels. Plasma and kidney AII values are depicted in Table II. On a per-weight basis, renal tissue exhibited more AII than did plasma. In comparison to control animals, plasma AII concentration among those receiving CsA was higher before glycine and lower during glycine infusion. Glycine appeared to decrease plasma AII concentrations in CsA-treated, but not control animals.

While there was a subtle main tendency for CsA to increase plasma AII, there was a strong main tendency for CsA to decrease the concentration of AII in renal tissue. These effects of CsA were not affected by L-arginine in the absence of glycine. Glycine infusion was associated with a major increase in kidney AII which obscured the effect of CsA in animals not receiving L-arginine. Among those receiving glycine, however, L-arginine feeding led to a further increment in kidney AII among $\mathrm{CON}$ rats, such that the effect of CsA reemerged. 
Table I. Micropuncture Results

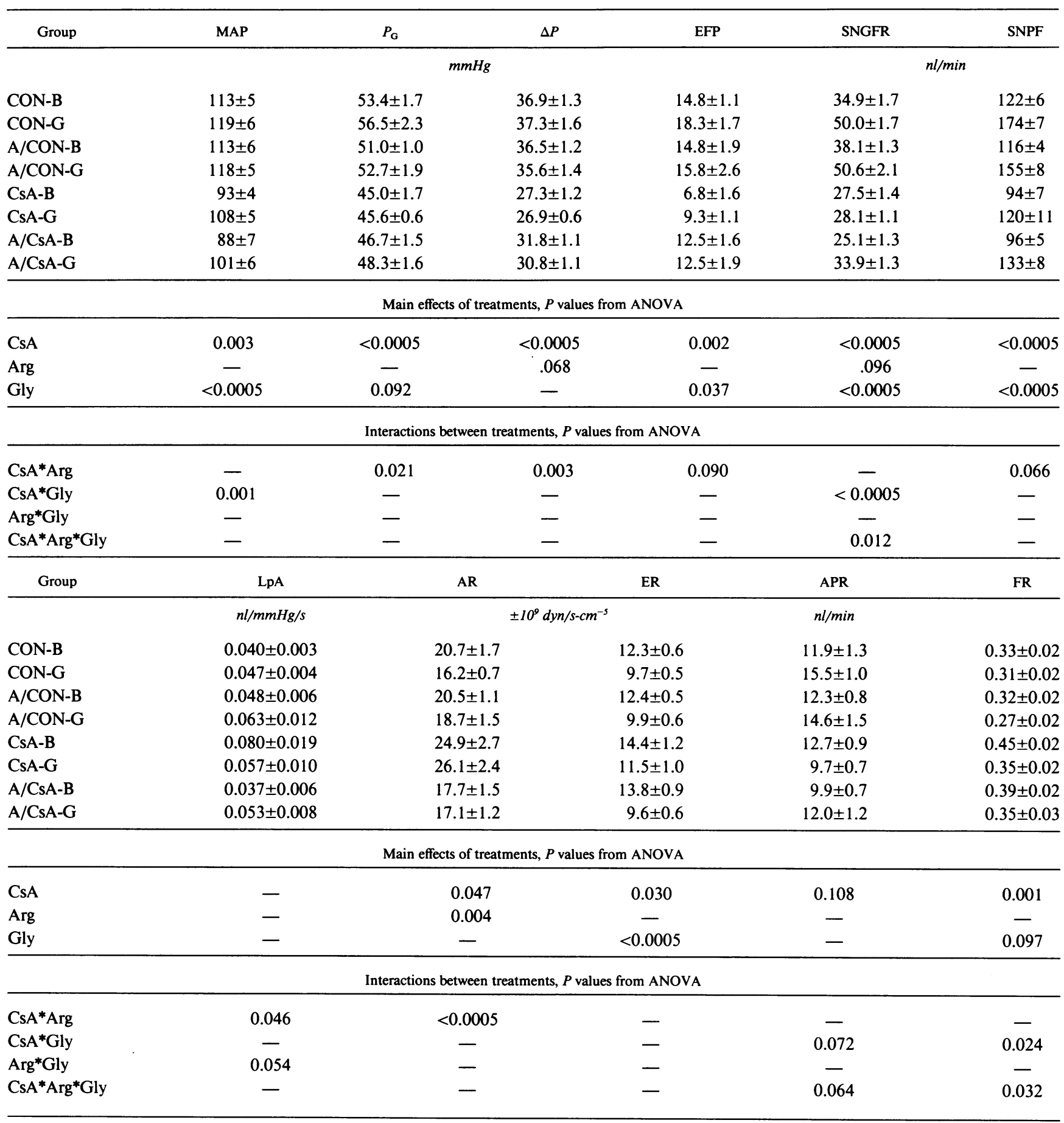

Abbreviations: CON, non-CsA; A/, L-arginine treated; B, before glycine infusion; G, during glycine infusion. Refer to text for other definitions. Probabilities referred to as "main effects" are those associated with a specific treatment independent of other treatments. An "interaction between treatments" refers to the probability of one treatment affecting the response to another, i.e., "CsA*Arg" refers to the probability that treatment with L-arginine conditions the response to CsA, etc.

\section{Discussion}

We previously reported that the functional nephrotoxicity of chronically administered CsA is mediated by a major decline in effective filtration pressure and a minor decrement in nephron plasma flow, each the consequence of uncompensated preglomerular vasoconstriction (6). These findings have been confirmed by others ( 7 ) and are reproduced in the present studies. A number of control systems are known to participate in maintaining a proper balance between pre- and postglomerular resis- 
tances, and the present studies provide evidence that CsA interferes with at least two of these influences. First, CsA interferes with normal endothelium-dependent relaxation of the afferent arteriole. Second, CsA appears to interfere with the compensatory generation of AII within the kidney, a response which is required to maintain efferent arteriolar tone and protect glomerular capillary hydrostatic pressure.

The notion that CsA nephrotoxicity involves damage to the vascular endothelium has been posed by others (27). Zoja et al. (8) reported time- and dose-dependent alterations in bovine endothelial cells exposed to CsA in culture. Incubation with CsA impairs endothelium dependent relaxation in response to acetylcholine in isolated resistance vessels from humans (28) and CsA treatment impairs subsequent ex vivo vasodilatory response to acetylcholine in arteries from rats (9-11). Kon et al. (27) reported that renal vasoconstriction after acute infusion of CsA in the rat was prevented by prior administration of an antibody to the endothelium-derived vasoconstrictor, endothelin (27). The present study adds to this body of knowledge by providing in vivo evidence for impaired renal activity of the endothelium dependent relaxing factor and natural antagonist to endothelin and AII, nitric oxide (NO). This evidence is manifested $(a)$ by afferent arteriolar vasoconstriction which is prevented by dietary supplementation with the NO substrate, L-arginine, (b) a diminished glomerular hemodynamic response to glycine infusion, and $(c)$ restoration of the response to glycine by treatment with L-arginine.

Arima et al. (29) has reported that an intact NO system exerts a greater influence over the afferent than the efferent glomerular arteriole. A depressed glomerular capillary hydro-

Table II. Plasma and Kidney AII Concentrations in Control Group (CON), Control Rats Treated with Arginine (A/CON), Cyclosporine Group (CsA), and Cyclosporine Rats Treated with Arginine $(A / C s A)$

\begin{tabular}{|c|c|c|c|c|}
\hline \multirow[b]{2}{*}{ Group } & \multicolumn{2}{|c|}{ Without glycine } & \multicolumn{2}{|c|}{ With glycine } \\
\hline & Plasma AII & Kidney AII & Plasma AII & Kidney AII \\
\hline & $p g / m l$ & $p g / g$ of kidney & $p g / m l$ & $p g / g$ of kidney \\
\hline $\mathrm{CON}$ & $46 \pm 12$ & $1217 \pm 186$ & $43 \pm 8$ & $2759 \pm 837^{*}$ \\
\hline $\mathrm{A} / \mathrm{CON}$ & $34 \pm 4$ & $889 \pm 168$ & $53 \pm 17$ & $5115 \pm 765^{*}$ \\
\hline CsA & $88 \pm 20$ & $694 \pm 114$ & $33 \pm 2$ & $2611 \pm 329^{*}$ \\
\hline $\mathrm{A} / \mathrm{CsA}$ & $82 \pm 14$ & $555 \pm 78$ & $32 \pm 3$ & $2424 \pm 746^{*}$ \\
\hline \multicolumn{2}{|c|}{$\begin{array}{l}\text { ANOVA table } \\
\text { main effects }\end{array}$} & \multicolumn{2}{|c|}{$\begin{array}{l}\text { Plasma AII } \\
P \text { value }\end{array}$} & $\begin{array}{c}\text { Kidney AII } \\
P \text { value }\end{array}$ \\
\hline \multirow{2}{*}{\multicolumn{2}{|c|}{$\begin{array}{l}\text { CsA } \\
\text { ARG }\end{array}$}} & \multirow{2}{*}{\multicolumn{2}{|c|}{0.031}} & 0.006 \\
\hline & & & & \\
\hline \multicolumn{2}{|l|}{ Glycine } & \multicolumn{2}{|c|}{0.002} & $<0.0005$ \\
\hline \multicolumn{5}{|c|}{ Interactions } \\
\hline \multicolumn{2}{|c|}{ CsA*ARG } & \multirow{2}{*}{\multicolumn{2}{|c|}{$<0.0005$}} & 0.019 \\
\hline \multirow{2}{*}{\multicolumn{2}{|c|}{$\begin{array}{l}\text { CsA*Glycine } \\
\text { ARG*Glycine }\end{array}$}} & & & \\
\hline & & & 0.054 \\
\hline \multicolumn{2}{|c|}{ CsA*ARG*Glycine } & \multicolumn{2}{|c|}{0.033} & 0.010 \\
\hline
\end{tabular}

Values not shown for $P \gg 0.05$. $^{*}$ By Student's $\mathrm{t}$ test, $P<.05$ vs. without glycine. static pressure which results from CsA administration is, therefore, compatible with the hypothesis that CsA interrupts the NO system. As a substrate for NO synthase, L-arginine, has been demonstrated to restore the NO system when that system has been damaged by disease $(10,17,19)$ or inhibited pharmacologically (30). Likewise, L-arginine feeding normalized the relationship between pre- and postglomerular resistances in animals treated with CsA, nearly restoring EFP to control levels, a finding consistent with a restoration of $\mathrm{NO}$ activity in this group. The renal vasodilatory response to amino acid infusion has been commonly employed as a test of renal functional reserve (31), and has been linked directly to NO in studies employing NO-synthase inhibition $(12,32,33)$. In the present study, CsA abolished the increase in SNGFR induced by glycine, but glycine-responsiveness was restored by L-arginine. These events would be expected if the effects of glycine were mediated by NO, and if the activity of NO was decreased by CsA and restored by $\mathrm{L}$-arginine. Despite the above observations, and despite a close resemblance between the A/CsA-B and CON-B groups with respect to the determinants of SNGFR, glycine-stimulated values of EFP, SNPF, and SNGFR in CON-G exceeded the respective values in A/CsA$G$, suggesting that not all of the glomerular hemodynamic effects of CsA are reversed by dietary supplementation with NO substrate. The mechanism by which CsA modifies NO activity remains unclear. CsA can decrease NO generation or accelerates NO degradation. The experimental maneuvers used in our study do not allow us to differentiate between these two possibilities and open a new area for further experimentation.

The present studies also suggest that CsA interrupts the relationship of plasma to kidney AII concentration. The role of the renin-angiotensin system in CsA nephrotoxicity has been a subject of controversy (6). Barros originally observed an increase in $\Delta P$ with decreases in LpA and SNPF in accounting for the decline in SNGFR which occurred in response to a single intravenous bolus of CsA, a pattern strikingly similar to that observed with AII infusion (34). It has been further suggested that the effects of CsA in this model could be partially prevented by angiotensin converting enzyme inhibition with captopril $(34,35)$. Antithetically, our findings in this short-term chronic model of CsA nephrotoxicity suggest that CsA administration resulted in a relative state of intrarenal AII deficiency, resulting in a failure of the efferent arteriole to compensate for preglomerular vasoconstriction, leading to a major decline in EFP and, subsequently, SNGFR. Indeed, by direct measurements, the present studies have demonstrated that CsA suppresses the quantity of AII in renal tissue independent of plasma AII concentration. However, since glycine infusion substantially increased kidney AII in all groups while its glomerular hemodynamic effects were blunted in CsA rats, the glomerular hemodynamic derangements resulting from CsA administration cannot be attributed solely to AII deficiency.

Loss of the vasodilatory response to glycine has been demonstrated in other models of renal injury in the rat including two-kidney one-clip Goldblatt hypertension (31), early diabetes mellitus (36), and experimental glomerulonephritis (37). In each of these cases, glycine infusion has been linked to a paradoxical decline in proximal tubular reabsorption, raising the possibility that the afferent arteriolar relaxation which usually results from glycine infusion was neutralized by a concurrent increase in delivery of fluid to the distal nephron with 
ensuing activation (or disinhibition) of the tubuloglomerular feedback (TGF) system. Consistent with this theme, Woods et al. have reported that renal functional reserve is impaired in dogs with experimental Fanconi syndrome or when TGF activity is diminished by a loop diuretic $(38,39)$. This pattern of glomerular and tubular response to glycine infusion also occurs in normal rats treated with the NO synthase inhibitor, LNMMA (12), suggesting that NO inhibition per se interferes with the normal tubular response to glycine. Interestingly, CsA nephrotoxicity provides another example in which the failure of glycine to induce renal vasodilation is accompanied by a decrease in proximal reabsorption. Furthermore, $\mathrm{L}$-arginine restores the tubular as well as glomerular hemodynamic response to glycine in this model of CsA nephrotoxicity.

Although inhibition of TGF and stimulation of NO have been previously discussed, the precise mechanism whereby glycine infusion leads to renal vasodilation has not been fully elucidated. We have previously observed that the administration of competitive antagonists to AII normalizes the glomerular and tubular responses to glycine during treatment with LNMMA (12), suggesting that NO normally functions within the proximal nephron to antagonize the anti-reabsorptive effects of AII (40). Armed with the new knowledge that glycine increases intrarenal AII concentration, we now propose that infusion of this substance induces parallel increases in NO and AII activity with the glomerular hemodynamic outcome contingent upon the net balance between the two systems. When the balance favors NO, proximal reabsorption increases, TGF activity is minimized, vasodilation ensues, and GFR increases. When the balance favors AII, proximal reabsorption declines, TGF is diminished and the vasodilatory influence of NO is overcome.

In conclusion, the present studies demonstrate that glycine infusion, while a stimulus to NO, also causes tissue AII levels to increase, that CsA nephrotoxicity is associated with decreased quantities of angiotensin II within renal tissue, and that dietary supplementation with L-arginine restores the glomerular and tubular responses to glycine infusion after short-term CsA treatment. CsA causes preglomerular vasoconstriction mediated in part by inadequate NO activity, and a decline in effective filtration pressure mediated in part by an inadequate AII response to glomerular hypotension. While glycine induces an increase in renal AII concentration, a normal NO response to glycine is inhibited by CsA such that no increase in GFR occurs. A pathogenetic role for abnormal nitric oxide activity in this in vivo model of chronic CsA nephrotoxicity is thus suggested.

\section{Acknowledgments}

These studies were supported by funds provided by the Research Service of the Department of Veterans Affairs and the National Institutes of Health (HL-37716). Dr. Luca De Nicola was recipient of a fellowship by National Kidney Foundation of Southern California.

\section{References}

1. Myers, B. D., J. Ross, L. Newton, J. Luetscher, and M. Perloth. 1984. Cyclosporine-associated chronic nephropathy. N. Engl. J. Med. 31 1:699-705.

2. Conte, G., A. Dal Canton, M. Sabbatini, P. Napodano, L. De Nicola, G Gigliotti, G. Fuiano, A. Testa, C. Esposito, D. Russo, et al. 1989. Acute cyclospor- ine renal dysfunction reversed by dopamine infusion in healthy subjects. Kidney Int. 36:1086-1089.

3. Murray, B. M., M. S. Paller, and T. F. Ferris. 1985. Effect of cyclosporine administration on renal hemodynamics in conscious rats. Kidney Int. 28:767774.

4. Sabbatini, M., C. Esposito, F. Uccello, L. De Nicola, M. Alba, G. Conte, A. Dal Canton, and V. E. Andreucci. 1988. Acute effects of cyclosporine on glomerular dynamics: micropuncture study in the rat. Transplant. Proc. 20 (Suppl. 3):544-548

5. Kaskel, F. J., P. Devarajan, L. A. Arbeit, J. S. Partin, and L. C. Moore. 1987. Cyclosporine nephrotoxicity: sodium excretion, autoregulation, and angiotensin II. Am. J. Physiol. 252:F733-F742.

6. Thomson, S. C., B. J. Tucker, F. B. Gabbai, and R. C. Blantz. 1989. Functional effects on glomerular hemodynamics of short-term chronic cyclosporine in male rats. J. Clin. Invest. 83:960-969.

7. Sabbatini, M., L. De Nicola, F. Uccello, G. Romano, G. Papaccio, V. Esposito V, V. Sepe, G. Conte, and G. Fuiano. 1991. Medium-term cyclosporine renal dysfunction and its reversibility in rats. Am. J. Physiol. 260:F898-F905.

8. Zoja, C., L. Furci, F. Ghilardi, P. Zilio, A. Benigni, and G. Remuzzi. 1986. Cyclosporine-induced endothelial cell injury. Lab. Invest. 55:455-462.

9. Yang, Z., D. Diederich, F. R. Buhler, and T. F. Luscher. 1989. Cyclosporine therapy impairs endothelium-dependent relaxations in the renal circulation. Kidney. Int. 35:511. (Abstr.)

10. Caramelo, C., A. Lopez Ferre, M. J. Gallego, S. Grandes, A. Riesco, R. Bosch, S. Casado, and L. Hernando. 1990. Cyclosporine A inhibits the endothelium-dependent, cGMP-mediated natriuretic, diuretic and vasodilatory responses: reversibility by L-arginine. J. Am. Soc. Nephrol. 1:608. (Abstr.)

11. Gallego, M. J., A. L. Garcia-Villalon, S. Grandes, J. L. Garcia, A. Lopez Farre, B. Gomez, S. Casado, G. Dieguez, L. Hernando, and C. Caramelo. 1991. Cyclosporine A treatment affects endothelium-dependent vasorelaxation, calcium uptake and platelet adhesion. J. Am. Soc. Nephrol. 2:662. (Abstr.)

12. De Nicola, L., R. C. Blantz, and F. B. Gabbai. 1992. Nitric oxide and angiotensin II: glomerular and tubular interaction in the rat. J. Clin. Invest. 89:1248-1256.

13. Nitta, K., A. L. Friedman, A. D. Nicastri, S. Paik, and E. A. Friedman. 1987. Granular juxtaglomerular cell hyperplasia caused by cyclosporine. Transplantation. 44:417-421.

14. Baxter, C. R., G. G. Duggin, B. M. Hall, J. S. Horvath, and D. J. Tiller. 1984. Stimulation of renin release from rat renal cortical slices by cyclosporine A. Res. Commun. Chem. Pathol. Pharmacol. 43:417-423.

15. Kurtz, A., R. Della Bruna, and K. Kuhn. 1988. Cyclosporine A enhances renin secretion and production in isolated juxtaglomerular cells. Kidney Int. 33:947-953.

16. Yuan, C. M., M. B. Pamnani, M. Abderlhaihm, and M. Payne. 1991. Effects of oral arginine on blood pressure in renin dependent hypertension in rats. J. Am. Soc. Nephrol. 2:486. (Abstr.)

17. Chen, P. Y., and P. W. Sanders. 1991. L-Arginine abrogates salt-sensitive hypertension in Dahl/Rapp rats. J. Clin. Invest. 88:1559-1567.

18. Chen, P. Y., and P. W. Sanders. 1991. The inducible form of nitric oxide synthase may participate in salt-sensitive hypertension in DAHL/RAPP rats. $J$. Am. Soc. Nephrol. 2:502. (Abstr.)

19. Reyes, A. A., M. L. Purkerson, I. Karl, and S. Klahr. 1992. Dietary supplementation with L-arginine ameliorates the progression of renal disease in rats with subtotal nephrectomy. Am. J. Kidney Dis. 20:168-176.

20. Marletta, M. A. 1989. Nitric oxide: biosynthesis and biological signifcance. Trends Pharmacol. Sci. 14:488-492.

21. Blantz, R. C., and B. J. Tucker. 1978. Measurement of glomerular dynamics. In Methods Pharmacol. (Renal Pharmacol.) 4B:141-163.

22. Blantz, R. C., L. C. Gushwa, C. A. Mundy, O. W. Peterson, and M. J. Ziegler. 1985. An examination of chronic angiotensin converting enzyme inhibition in the rat. Miner. Electrolyte Metab. 11:249-255.

23. Lowry, O. H., N. J. Rosebrough, A. L. Farr, and R. J. Randall. 1951. Protein measurement with the Folin Phenol reagent. J. Biol. Chem. 193:265275.

24. Landis, E. M., and R. J. Pappenheimer. 1963. Exchanges of substances through capillary walls. In Handbook of Physiology, Circulation (Sect. 2, Volume II). American Physiological Society, Washington, DC. 931-1034.

25. Blantz, R. C. 1974. Effect of mannitol upon glomerular ultrafiltration in the hydropenic rat. J. Clin. Invest. 54:1135-1143. IL.

26. Wilkinson, L. 1989. Systat: the System for Statistics. Systat Inc., Evanston,

27. Kon, V., M. Sugiura, T. Inagami, B. R. Harvie, I. Ichikawa and R. L. Hoover. 1990. Role of endothelin in cyclosporine induced glomerular dysfunction. Kidney Int. 37:1487-1491.

28. Richards, N. T., L. Poston, and P. J. Hilton. 1989. Cyclosporine A inhibits relaxation but does not induce vasoconstriction in human subcutaneous resistance vessels. J. Hypertens. 7:1-3. 
29. Arima, S., L. A. Juncos, Y. Ren, O. A. Carretero, and S. Ito. 1992. Endothelium derived relaxing factor/nitric oxide accounts for different sensitivity to angiotensin II in microperfused rabbit afferent but not efferent arterioles (abstract). J. Am. Soc. Nephrol. 3:539.

30. Moncada, S., R. M. J. Palmer, and E. A. Higgs. 1991. Nitric oxide: physiology, pathophysiology and pharmacology. Pharmacol. Rev. 43:109-142.

31. De Nicola, L., R. C. Blantz, and F. B. Gabbai. 1991. Renal functional reserve in treated and untreated hypertension. Kidney Int. 40:406-412.

32. Tolins, J. P., and L. Raij. 1991. Effects of amino acid infusion on renal hemodynamics: role of endothelial-derived relaxing factor. Hypertension. 17:1045-1051.

33. King, A. J., J. L. Troy, S. Anderson, J. R. Neuringer, M. Gunning, and B. M. Brenner. 1991. Nitric oxide; a potential mediator of amino acid-induced renal hyperemia and hyperfiltration. J. Am. Soc. Nephrol. 1:1271-1277.

34. Barros, E. J. M., M. A. Boim, H. Ajzen, O. L. Ramos, and N. Schor. 1987. Glomerular hemodynamics and hormonal participation on cyclosporine nephrotoxicity. Kidney Int. 32:19-25.
35. Bantle, J. P., M. S. Paller, R. J. Boudreau, M. T. Olivair, and T. F. Ferris. 1990. Long term effects of cyclosporine on renal function in organ transplant recipients. J. Lab. Clin. Med. 115:233-240.

36. De Nicola, L., R. C. Blantz, and F. B. Gabbai. 1992. Renal functional reserve in the early stage of experimental diabetes. Diabetes. 41:267-273.

37. De Nicola, L., C. B. Wilson, J. A. Keiser, R. C. Blantz, and F. B. Gabbai. 1991. Effect of angiotensin converting enzyme inhibition on renal functional reserve in rats with chronic glomerulonephritis with and without hypertension. $J$. Am. Soc. Nephrol. 2:517. (Abstr.)

38. Woods, L. L., and E. W. Young. 1991. Impaired renal hemodynamic response to protein feeding in dogs with experimental Fanconi syndrome. Am.J. Physiol. 261:F14-F21

39. Woods, L. L., D. R. De Young, and B. E. Smith. 1990. Furosemide abolishes the increase in glomerular filtration rate after a meat meal (abstract). FASEB J. 4:A437.

40. Harris, P. F., and J. A. Young. 1977. Dose-dependent stimulation and inhibition of proximal tubular sodium reabsorption by angiotensin II in the rat kidney. Pflügers Arch. Eur. J. Physiol. 367:295-297. 